\title{
Fatigue in patients with colorectal cancer treated with capecitabine and oxaliplatin
}

Sá Lilian A ${ }^{1}$, Vettori Josiane $C^{1}$, Moreira Cláudia LR ${ }^{1}$, Leandro Miraya $\mathrm{S}^{1}$, Tirapelli Daniela PC ${ }^{2}$, Perdoná Gleici SC ${ }^{3}$, Souza Hayala $\mathrm{CC}^{1}$, Oliveira Harley F${ }^{4}$, Santos Fabiana N ${ }^{1}$, Rapatoni Liane ${ }^{1}$, Ribeiro Karen B ${ }^{1}$, Almeida Thiago CL', Feres Omar ${ }^{2}$, Rocha José JRR ${ }^{2}$ and Peria Fernanda $\mathrm{M}^{3 *}$

${ }^{1}$ School of Medicine of Ribeirão Preto,University of São Paulo, Brazil

${ }^{2}$ Department of Surgery and Anatomy, School of Medicine of Ribeirão Preto, University of São Paulo, Brazil

${ }^{3}$ Department of Social Medicine, School of Medicine of Ribeirão Preto, University of São Paulo, Brazil

${ }^{4}$ Department of Internal Medicine, School of Medicine of Ribeirão Preto, University of São Paulo, Brazil

\begin{abstract}
Cancer-related fatigue is characterized by feelings of extreme tiredness, which do not improve with rest and negatively affects the physical and psychosocial function of patients, significantly reducing their quality of life. The prevalence of fatigue in cancer patients undergoing chemotherapy can be as high as $90 \%$. Colorectal cancer is one of the neoplasms with the highest incidence of cancer-related fatigue, especially among individuals older than 65 years old. To perform a comparative assessment ofcancer-related fatigue in patients with colorectal cancer during the first four cycles of chemotherapy using the CAPOX (capecitabine and oxaliplatin) regimen.

From 35 individuals initially recruited, 27 responded to the FACT-F (Functional Assessment of Chronic Illness Therapy - Fatigue scale) questionnaire at the four timepoints stipulated to assess fatigue. The average age of the sample population was 61.9 (33-84) years old, and most participants were female (66.7\%). Analysis of the data revealed that fatigue did not increase and that the quality of life did not decrease over the course of chemotherapy using CAPOX. Analysis of the confidence interval of the difference of the means for the qualitative variables of theFACT-F (fatigue) and FACT-G (quality of life) questionnairesrevealed that gender was the only variable that exhibited a significant correlation with fatigue, as the scores of females were correlated with greater fatigue compared with the males ( $\mathrm{p}=0.03$ ). Fatigue did not increase in patients with colorectal cancerduring the course of the first four cycles of chemotherapy using the CAPOX regimen. The results indicated that fatigue was stronger among females compared with males.
\end{abstract}

\section{Introduction}

Cancer-related fatigue is characterized by feelings of severe tiredness that do not improve with rest and significantly impair the quality of life of patients in various stages of cancer [1]. Despite being considered one of the worse symptoms and occurring frequently, fatigue is not always given adequate attention by healthcare professionals orthe patients themselves [2].

Fatigue may be present at the time of diagnosis, and its prevalence exhibits a remarkable increase as the disease progresses $[3,4]$. The prevalence of cancer-related fatigue may be as high as $75 \%$ in untreated patients and may reach $90 \%$ among patients undergoing radiotherapy and/or chemotherapy $[5,6]$.

The incidence and prevalence of colorectal cancer are high worldwide, and individuals older than 60 years old are the most affected. Relapse occurs in approximately $40 \%$ of the patients with nonmetastatic disease at diagnosis who are subjected to curative surgery $[7,8]$.

The literature describes several instruments for the diagnosis and measurement of fatigue intensity, among which the Functional Assessment of Cancer Therapy -Fatigue Scale (FACT-F) stands out. The FACT-F is a 40-item unidimensional questionnaire that allows detection of significant variations in the degree of fatigue and correlation of these variations with the various treatments used in cancer and their outcomes. In addition, the FACT-F has already been translated and validated for the Brazilian population [9-12].

Fatigue poses a major challenge for oncology, as few clinical studies have investigated specific treatments for this symptom. Its pathogenesis is unknown, butfatigue is assumed to be multifactorial, involving the participation of psychological, social, and physiological components. Among the latter, anemia, disorders of the hypothalamicpituitary-adrenal axis, serotonin metabolism disorders, and increased proinflammatory cytokine activity have been investigated. Proinflammatory cytokines may represent the common denominator underlying the various pathways involved in cancer and either in fatigue development $[13,14]$.

As colorectal tumors are one of the most prevalent forms of neoplasia and given that a high percentage of oncology patients

Correspondence to: Fernanda Maris Peria, Avenida dos Bandeirantes, 3900 Campus USP, Hospital das Clinicas, Departamento de Clinica Médica, $6^{\circ}$ andar, Bairro Monte Alegre, Ribeirão Preto, São Paulo, Brazil, Tel: +55 16 36325978; E-mail: fernandaperia@fmrp.usp.br

Key words: fatigue, quality of life, colorectal cancer, chemotherapy, FACT-F

Received: January 10, 2015; Accepted: January 23, 2015; Published: January 27, 2015 
experience fatigue at some point during treatment, that quality of life significantly decreases due to fatigue, and that the pathophysiology of fatigue remains poorly understood, studies aimed at assessing the impact of fatigue on oncology patients are needed to contribute to the development of possible therapies and clinical interventions.

Therefore, the aim of the present study was to assess the presence of fatigue in patients with colorectal cancer before the first four cycles of chemotherapy using the CAPOX protocol (capecitabine and oxaliplatin) by the FACT-F questionnaire and to assess the progression of this symptom over the course of subsequent chemotherapy cycles.

\section{Materials and methods}

From January to August of 2014, 35 patients diagnosed with colorectal cancer and subjected to treatment with the CAPOX regimen as outpatients at the chemotherapy unit of the Teaching Hospital of the Medical School of RibeirãoPreto (Hospital das Clínicas da Faculdade de Medicina de RibeirãoPreto - HC-FMRP/USP) were recruited for the present study. Only 27 participants filled out the questionnaires at the four stipulated timepoints and were included in the analysis.

To analyze the presence of fatigue at baseline and its progression during the four first cycles of chemotherapy, the participants were asked to answer all of the questions listed in FACT-F, which investigates the physical, social/family, emotional, and functional wellbeing domains, in addition to a section specifically focused on the assessment of fatigue. The participants' total scores were then calculated.

The answers to the FACT-F items were also used to calculate the scores of FACT-G, which provides a measure of the patients' quality of life.

The inclusion criteria were as follows: patients diagnosed with metastatic colorectal cancer beginning first-line or adjuvant chemotherapy with the CAPOX regimen. The exclusion criteria were as follows: cognitive deficits that prevented the participants from understanding the experimental design and from answering the FACT-F questionnaire, brain metastasis, and the use of corticoids or anti-inflammatory agents.

Statistical analysis to assess the correlation between the investigated variables and scores was based on descriptive and association techniques. Linear regression analysis was performed to investigate the functional relationships among the variables.

The study was subjected to and approved by the Research Ethics Committee of HC-FMRP/USP, no. 11755/ 2010.

\section{Results}

Of the 27 individuals included in the study, 16 and 11 patients underwent adjuvant and palliative first-line chemotherapy, respectively. The average age of the sample population was 61.92 years old, varying from 33 to 84 years old. Most participants (59.26\%) were 60 years old or older. No correlation was observed between the type of chemotherapy indication and the patients' age.

Females were predominant in the present sample (66.67\%), 13 participants were married (48.14\%), and more than half had attended up to elementary school. Eight different occupations were mentioned by the participants, and $48.14 \%$ were retired.

Regarding the family history of cancer, the number of positive and negative answers was identical, i.e., 13, and one participant did not answer this item.
The tumor was located in the colon in 13 participants (48.15\%) and in the rectum in the remainder of the sample. All of the patients were in stage III $(59.3 \%)$ or IV $(40.7 \%)$, and only seven exhibited distant metastases (25\%).

No participant required protocol changes or used corticoids or antidepressants. Only one patient exhibited a hemoglobin concentration $<10.0 \mathrm{~g} / \mathrm{dL}$; this patient's hemoglobin was $9.9 \mathrm{~g} / \mathrm{dL}$ at the second chemotherapy cycle.

The average score in the fatigue questionnaire was 114.20 , and the average overall score (quality of life) was 76.50. The lowest score among the four chemotherapy cycles was usedfor each individual. The scores obtained from the questionnaires were similar regardless of the chemotherapy cycle (Table 1), thereby indicating that fatigue did not increase over the course ofthefour chemotherapy cycles using the CAPOX regimen $(p=0.2574)$.

The FACT-G scores remained stable (Table 2), thus indicating that the participants' quality of life did not become worse over the course of treatment $(p=0.2411)$.

The four main subscales of the FACT-F (physical wellbeing, social/ family wellbeing, emotional wellbeing and functional wellbeing) and the fatigue-specific subscale were analyzed separately, but no differences were identified among the four cycles of chemotherapy.

Similarly, the FACT-F-TOI scores, which comprise all the domains in FACT-G, except for emotional wellbeing (Table 3), were also analyzed, but no difference was detected among the four cycles of chemotherapy $(p=0.6461)$.

To assess the possible correlation between the scores and variables, Pearson's coefficient was calculated. Relative to the variable age, the results indicated a weak correlation with the FACT-F and FACT-G scores.

The average FACT-F scores per tumor site were quite similar: 110 for patients with colon involvement and 117 for patients with rectal

Table 1. Descriptive summary of the scores of the FACT-F questionnaire (Friedman chisquared $=4.0376, \mathrm{df}=3, p=0.2574$ ).

\begin{tabular}{|c|c|c|c|c|c|c|c|c|}
\hline Cycle & Min. & $\mathbf{1}^{\text {st }} \mathbf{Q}$ & Median & Mean & $\mathbf{3}^{\text {rd }} \mathbf{Q}$ & Max & Missing & SD \\
\hline $1^{\text {st }}$ & 87.66 & 118.30 & 135.00 & 131.50 & 143.90 & 152.70 & 2.00 & 15.88 \\
\hline $2^{\text {nd }}$ & 99.83 & 119.70 & 129.00 & 127.70 & 141.20 & 149.00 & 2.00 & 14.86 \\
\hline $3^{\text {rd }}$ & 89.33 & 111.80 & 128.20 & 125.90 & 140.80 & 158.00 & 2.00 & 18.07 \\
\hline $4^{\text {th }}$ & 29.5 & 115.5 & 132.3 & 125.2 & 144.9 & 160.0 & 2.00 & 28.85 \\
\hline
\end{tabular}

Table 2. Descriptive summary of the scores of the FACT-G questionnaire (Friedman chisquared $=4.1955, \mathrm{df}=3, p=0.2411)$.

\begin{tabular}{|c|c|c|c|c|c|c|c|}
\hline Cycle & Min. & $\mathbf{1}^{\text {st }}$ & Median & Mean & $\mathbf{3}^{\text {rd }}$ & Max. & SD \\
\hline 1 & 58.66 & 77.00 & 89.16 & 87.08 & 96.08 & 102.80 & 11.78 \\
\hline 2 & 64.10 & 79.00 & 88.00 & 85.42 & 93.83 & 101.00 & 10.51 \\
\hline 3 & 58.16 & 77.12 & 86.00 & 84.70 & 94.63 & 107.00 & 12.25 \\
\hline 4 & 17.50 & 72.33 & 90.83 & 84.05 & 96.74 & 108.00 & 19.53 \\
\hline
\end{tabular}

Table 3. Descriptive summary of the scores of theFACT-F-TOI questionnaire (Friedman chi-squared $=1.6591, \mathrm{df}=3, p=0.6461$ ).

\begin{tabular}{|c|c|c|c|c|c|c|c|}
\hline Cycle & Min. & $\mathbf{1}^{\text {st }} \mathbf{Q}$ & Median & Mean & $\mathbf{3}^{\text {rd }} \mathbf{Q}$ & Max. & SD \\
\hline 1 & 61.00 & 85.00 & 94.00 & 89.44 & 98.50 & 104.00 & 11.70 \\
\hline 2 & 59.00 & 80.66 & 89.00 & 86.62 & 96.00 & 104.00 & 11.63 \\
\hline 3 & 53.00 & 73.50 & 89.00 & 84.43 & 94.46 & 106.00 & 14.23 \\
\hline 4 & 19.00 & 78.00 & 90.50 & 83.95 & 97.00 & 108.00 & 21.04 \\
\hline
\end{tabular}



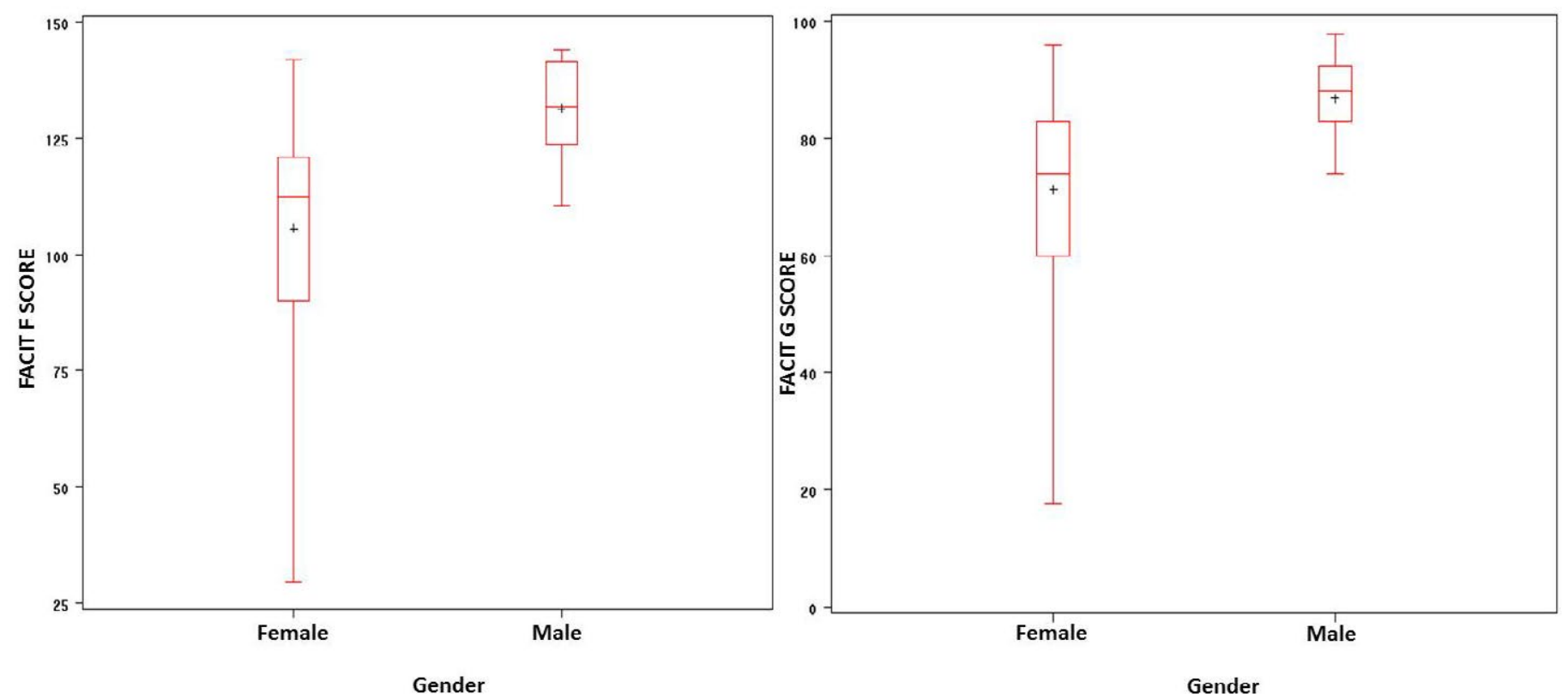

Figure 1. FACIT-F and FACIT-G scores and correlation with gender.

disease. The same was true for the FACT-G scores; the patients with colon tumors exhibited lower FACT-G scores. The same behavior was observed when the scores were analyzed as a function of the type of chemotherapy indication, family history of cancer, and tumor stage. Therefore, the average scores were quite similar, and the patients with palliative indication, positive family history of cancer, and stage IV exhibited lower scores.

Analysis of the scores according to gender revealed that males scored higher in both investigated scales and with little variability in the results. The average FACT-F score of the female participants was lower than the minimum male score (Figure 1). Similar behavior was observed upon comparing the participants with and without metastases; the scores from the male patients were lower on both scales.

Analysis of the scores according to educational level revealed that the average score of the participants with higher education was higher than the remainder of the patients, whereas the patients who attended only elementary school exhibited the lowest scores.

To investigate the correlation between the scores onthe FACT-F and FACT-G scales and the variables age, gender, tumor stage, type of chemotherapy indication, and tumor site, two linear regression models were fitted. Only gender exhibited a significant association with the fatigue scores $(p<0.05)$, as the average score in the FACT-F was 22 points higher for males than females. Gender also exhibited a significant correlation with the FACT-G scores, which were on average 12 points higher for males (Figure 1 ).

\section{Discussion}

Analysis of the data collected from the 27 participants comprising the sample population of the present study revealed that gender was the only variable that correlated with fatigue; the scores of the females were lower compared with the scores formales. Those findings are consistent with the results of a study that assessed the toxicity of a therapeutic protocol for colorectal cancer, which reported that the female participants exhibited lower fatigue scores [15].
According to the results of our study, the patients' quality of life remained constant throughout the course of treatment using the CAPOX regimen, and fatigue did not increase. We believe that these results are potentially associated with the participants' positive response to treatment, which allowed for control of the tumor progression. The drug regimen employed is another factor that must be taken into account, as the use of oral chemotherapy (capecitabine) is associated with better quality of life compared with regimens including fluorouracil infusion, which requires hospitalization. Some studies observed better quality of life in patients using CAPOX compared to FOLFOX, a similar regimen in which the medications are administered by infusion.[16]

The results of the present study, namely, the consistent quality of life and the unchanged level of fatigue, contradict the findings reported by other authors relative to other chemotherapy regimens; those authors observed correlations between systemic cancer treatment and the incidence of fatigue. According to such studies, the frequency of fatigue among oncology patients tends to increase during the course of chemotherapy and/or radiotherapy [17-20]. One study assessed 379 patients with a past history of chemotherapy for any type of cancer and found that $25 \%$ of the participants reported fatigue as the side effect with the greatest impact following the end of a cycle of chemotherapy. In addition, more than half of the sample noticed that fatigue lasted longer than any other symptom, including nausea, depression, and pain, which negatively impact the quality of life of such patients [21].

According to the results of the present study, the participants' quality of life did not become worse during chemotherapy, which might be due to the constant level of fatigue and possibly to the tumor response to cancer treatment. These findings are of paramount clinical importance, as the main goal of palliative treatment is to relieve symptoms. Within that context, a particular concern is to maintain the patients' quality of life as long as possible during the course of treatment and to minimize the adverse events and symptoms so that the patients might assess their treatment positively over the entire course of chemotherapy. In a recent study with 48 patients with colorectal cancer treated with 
5-fluorouracil and folinic acid in the outpatient setting, the participants reported improvement in their quality of life over the course of the experiment, which the authors attributed to their expectations relative to the end of chemotherapy [22].

Relevant results were also obtained in another study, where 78 patients with colorectal cancer subjected to an average of five to eight cycles of chemotherapy using 5-fluorouracil and eniluracil per oral route assessed their quality of life. At the end of treatment, significant improvements in the patients' overall quality of life were observed, in addition to specific improvements in the emotional domain compared with the assessment before treatment [23].

One additional study also reported satisfactory quality of life in patients with colorectal cancer subjected to adjuvant therapy. The results indicated a reduction in anxiety and improvements of physical, emotional, and social function over time [24].

One should bear in mind that cancer diagnosis and treatment exert a direct effect on the quality of life of patients with colorectal cancer. In addition, aspects such as an appropriate nutritional and cardiovascular state, practice of exercise, and the biopsychosocial structure of patients also contribute to their quality of life [25].

These data point to the difficulties in the measurements of the quality of life of patients with cancer; patient quality of life is affected by countless factors related to treatment and by the individual's life context.

The results of the present study demonstrate that the quality of life of the participants did not change and that fatigue did not increase during the first four cycles of chemotherapy using the CAPOX regimen in patients with colorectal cancer.

\section{Disclosure}

The present study was financed by the São Paulo Research Foundation (Fundação de Amparo à Pesquisa do Estado de São Paulo - FAPESP), process no. 2010/19961-2.

\section{Acknowledgement}

The authors thank Ms. Leida Mara de Lima Almeida for her help in the collection and organization of the data used in the present study.

\section{References}

1. Cella D, Lai JS, Chang CH, Peterman A, Slavin M (2002) Fatigue in cancer patients compared with fatigue in the general United States population. Cancer 94: 528-538. [Crossref]

2. Glaus A, Crow R, Hammond S (1996) A qualitative study to explore the concept of fatigue/tiredness in cancer patients and in healthy individuals. Eur J Cancer Care 5: 8-23. [Crossref]

3. Magnusson K, Möller A, Ekman T, Wallgren A (1999) A qualitative study to explore the experience of fatigue in cancer patients. Eur J Cancer Care (Engl) 8: 224-232. [Crossref]

4. Jean-Pierre P, Figueroa-Moseley CD, Kohli S, Fiscella K, Palesh OG, et al. (2007) Assessment of cancer-related fatigue: implications for clinical diagnosis and treatment. Oncologist 12: 11-21. [Crossref]

5. Hofman M, Ryan JL, Figueroa-Moseley CD, Jean-Pierre P, Morrow GR (2007) Cancerrelated fatigue: the scale of the problem. Oncologist 12: 4-10. [Crossref]

6. Prue G, Rankin J, Allen J, Gracey J, Cramp F (2006) Cancer-related fatigue: A critical appraisal. Eur J Cancer 42: 846-863. [Crossref]

7. Gryfe R, Kim H, Hsieh ET, Aronson MD, Holowaty EJ, et al. (2000) Tumor microsatellite instability and clinical outcome in young patients with colorectal cancer. N Engl J Med 342: 69-77. [Crossref]
8. INCA-Ministério da Saúde. Incidência de Câncer no Brasil 2009 [Incidence of cancer in Brazil]. Available from: http://www.inca.gov.br/estimativa/2010/index. asp?link=conteudo_view.asp\&ID=2. Accessed on July 4, 2010.

9. Yellen SB, Cella DF, Webster K, Blendowski C, Kaplan E (1997) Measuring fatigue and other anemia-related symptoms with the Functional Assessment of Cancer Therapy (FACT) measurement system. J Pain Symptom Manage 13: 63-74. [Crossref]

10. Bennett B, Goldstein D, Lloyd A, Davenport T, Hickie I (2004) Fatigue and psychological distress--exploring the relationship in women treated for breast cancer. Eur J Cancer 40: 1689-1695. [Crossref]

11. Tchen N, Juffs HG, Downie FP, Yi QL, Hu H, et al. (2003) Cognitive function, fatigue, and menopausal symptoms in women receiving adjuvant chemotherapy for breast cancer. J Clin Oncol 21: 4175-4183. [Crossref]

12. Carroll JK, Kohli S, Mustian KM, Roscoe JA, Morrow GR (2007) Pharmacologic treatment of cancer-related fatigue. Oncologist 12: 43-51. [Crossref]

13. Ahlberg K, Ekman T, Gaston-Johansson F, Mock V (2003) Assessment and management of cancer-related fatigue in adults. Lancet 362: 640-650. [Crossref]

14. Jager A, Sleijfer S, van der Rijt CC (2008) The pathogenesis of cancer related fatigue: could increased activity of pro-inflammatory cytokines be the common denominator? Eur J Cancer 44: 175-181. [Crossref]

15. Greenberg DB, Gray JL, Mannix CM, Eisenthal S, Carey M (1993) Treatment-related fatigue and serum interleukin-1 levels in patients during external beam irradiation for prostate cancer. J Pain Symptom Manage 8: 196-200. [Crossref]

16. Berger AM, Grem JL, Visovsky C, Marunda HA, Yurkovich JM (2010) Fatigue and other variables during adjuvant chemotherapy for colon and rectal cancer. Oncol Nurs Forum 37: E359-369. [Crossref]

17. Stasi R, Abriani L, Beccaglia P, Terzoli E, Amadori S (2003) Cancer-related fatigue evolving concepts in evaluation and treatment. Cancer 98: 1786-1801. [Crossref]

18. Curt GA, Breitbart W, Cella D, Groopman JE, Horning SJ, et al. (2000) Impact of cancer-related fatigue on the lives of patients: new findings from the Fatigue Coalition. Oncologist 5: 353-360. [Crossref]

19. Machado SM, Sawada NO (2008) Assessment of the quality of life of cancer patients using adjuvant chemotherapeutic treatment. TextoContextoEnferm, Florianópolis 17: 750-757.

20. Broeckel JA, Jacobsen PB, Balducci L, Horton J, Lyman GH (2000) Quality of life after adjuvant chemotherapy for breast cancer. Breast Cancer Res Treat 62: 141-150. [Crossref]

21. Haller DG, Tabernero J, Maroun J, de Braud F, Price T, et al. (2011) Capecitabine plus oxaliplatin compared with fluorouracil and folinic acid as adjuvant therapy for stage III colon cancer. J Clin Oncol 29: 1465-1471. [Crossref]

22. Chaves PL, Gorini MIPC (2011) Quality of life of patients with colorectal cancer treated with chemotherapy in the outpatient setting. Rev GaúchaEnfer, Porto Alegre (RS) 32: 767-773.

23. Ramsey SD, Andersen MR, Etzioni R, Moinpour C, Peacock S, et al. (2000) Quality of life in survivors of colorectal carcinoma. Cancer 88: 1294-1303. [Crossref]

24. Hobday TJ, Kugler JW, Mahoney MR, Sargent DJ, Sloan JA, et al. (2002) Efficacy and quality-of-life data are related in a phase II trial of oral chemotherapy in previously untreated patients with metastatic colorectal carcinoma. J Clin Oncol 20: 4574-4580. [Crossref]

25. Nicolussi AC, Sawada NO (2009) Quality of life of patients with colorectal cancer using adjuvant therapy. Acta Paul Enferm 22: 155-161.

26. Nicolussi AC, Sawada NO (2010) Factors influencing the quality of life of patients with colon and rectal cancer. Acta Paul Enferm 23: 125-130.

Copyright: (C) 2015 Sá Lilian A. This is an open-access article distributed under the terms of the Creative Commons Attribution License, which permits unrestricted use, distribution, and reproduction in any medium, provided the original author and source are credited. 